
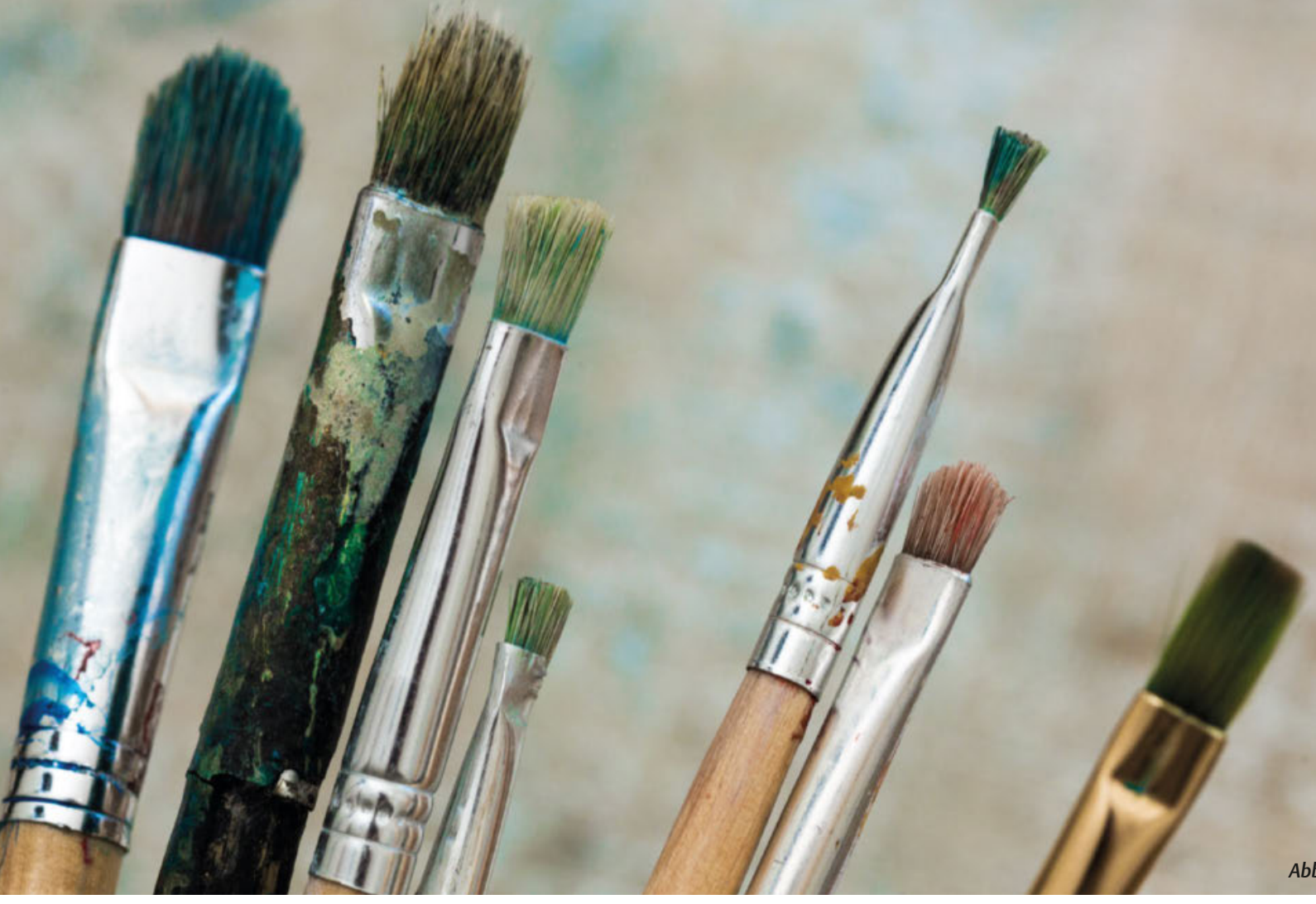

Abb. 1 (c) fotofabrika/stock.adobe

\title{
Kunsttherapie in der
}

Palliativversorgung.

\section{Ein narratives Review.}

\section{Teil I: Forschungsstand}

Durch bildende Kunst lässt sich die Lebensqualität von Palliativpatienten verbessern Diese Arbeit gibt einen Einblick in den Stand der Forschung

Ria Kortum, Sabine Koch und Harald Gruber

Kontext und Ausgangssituation dieser Übersichtsarbeit

Die Diskussion um die Gestaltung des Lebensendes, der Umgang mit Tod und Sterben und die damit verbundenen Belastungen und Herausforderungen hat in den letzten Jahrzehnten erheblich an Dynamik gewonnen. Im Zusammenhang mit der Überalterung der Gesellschaft, besseren Behandlungsmethoden schwerer Erkrankungen und der gleichzeitigen Zunahme chronischer Erkrankungen ist die
Frage des Umgangs mit dem Lebensende auch eine gesamtgesellschaftliche geworden [13]. Damit einhergehend hat sich die von Cicely Saunders und dem 1967 gegründeten St. Christophers Hospiz wesentlich geprägte Leitidee, lebensbedrohlich Erkrankten und Sterbenden mit mehr Respekt und Würde zu begegnen, weltweit verbreitet [20]. In Deutschland wurden erste Palliativstationen, die dieser Philosophie folgten, in den 1980er Jahren eröffnet. Mittlerweile ist der palliativmedizinische Ansatz in viele Bereiche des ge- 


\section{Zusammenfassung}

Kunsttherapie (Therapie mit bildnerischen Medien) wird in der Palliativversorgung seit über 30 Jahren angeboten und im Rahmen der palliativen Komplexbehandlung (OPS 9-982) neben anderen begleitenden Therapiemaßnahmen empfohlen. Entgegen einer breiten praktischen $\mathrm{Er}$ fahrung ist ihre Wirkung bisher jedoch wissenschaftlich kaum untersucht. Auf Basis einer systematischen Datenbankrecherche wurde der aktuelle Forschungs- und Erfahrungsstand ermittelt und in 2 Übersichtsarbeiten aufgeteilt. In dieser Publikation liegt der Schwerpunkt auf der Methodik zur Erstellung des Reviews und der Darstellung des Forschungsstands. Unter insgesamt 82 internationalen Artikeln konnten 5 an größeren Stichproben durchgeführte Studien gefunden werden, in denen sich positive Effekte von Kunsttherapie auf Depression, Traurigkeit, Ängste, Müdigkeit, Fatigue, Wohlbefinden, Krankheitsgefühl und Schmerz aufzeigen ließen. Eine Validierung dieser Befunde durch Kontrollgruppenstudien steht in der Mehrzahl der Fälle noch aus. Als erste positive Befunde sind diese Ergebnisse jedoch eine wichtige Grundlage für weitere Studien und damit zur Evidenzbasierung der Kunsttherapie in der Palliativversorgung.

sundheitlichen Versorgungssystems integriert und in Form von häuslicher Betreuung, stationären Angeboten (Palliativstation, Hospiz, Konsiliardienst) und ambulanten Diensten etabliert [34].

\section{Bedürfnisse von Palliativpatienten}

Als Palliativmedizin wird nach der Definition der Deutschen Gesellschaft für Palliativmedizin „die Behandlung von Patienten mit einer nicht heilbaren, progredienten und weit fortgeschrittenen Erkrankung mit begrenzter Lebenserwartung“ beschrieben, „für die das Hauptziel der Begleitung die Lebensqualität ist“ [35]. Unabhängig von der zu erwartenden Lebensdauer soll die Belastung durch die Erkrankung erträglich sein, um die letzte Zeit des Lebens so gut wie möglich zu gestalten. Wie aktuelle Studien aufzeigen, sind dies die zentralen Motive, aufgrund derer Patienten palliative Behandlungsangebote in Anspruch nehmen. Sie wünschen sich Symptomkontrolle und Schmerzreduktion, um ihr grundlegendes Bedürfnis nach Autonomie, Betätigung, Kreativität und sozialen Kontakten zu unterstützen [4, 23]. Es geht ihnen nicht primär darum, Sterbebegleitung in Anspruch zu nehmen, sondern entscheidend ist das Bedürfnis nach bestmöglicher Gestaltung der verbleibenden Lebenszeit [43].

\section{Kunsttherapeuten als Teil multidisziplinärer Teams}

Multidisziplinäre Teams werden zusammengesetzt, um möglichst umfassend physische, psychische, soziale und spirituelle Bedürfnisse der Patienten und die damit verbundenen multifaktoriellen Belastungsfaktoren und -reaktionen zu berücksichtigen [28]. Auch Kunsttherapie, d.h. Therapie mit bildnerischen Mitteln (z. B. Zeichnen, Malen, Plastizieren oder Fotografieren) gibt es in der Palliativversorgung seit über 30 Jahren. Sie wird gleichwertig mit Musiktherapie als „künstlerische Therapien (Kunst- und Musiktherapie)“ im
Rahmen der palliativmedizinischen Komplexbehandlung (OPS 9-982) als eine von 9 primär nicht-ärztlichen oder pflegerischen Tätigkeiten vorgeschlagen, von denen mindestens 2 Angebote als begleitende Behandlung eingesetzt werden sollen. Nach einer Befragung im Rahmen des Forschungsprojektes „Psychosoziale Begleitung auf Palliativstationen in Deutschland“ haben 40,7\% der Palliativstationen sowie 15,5\% der stationären Hospize einen Kunsttherapeuten Kunsttherapeutin fest im Team integriert. 11,2\% (Palliativstationen) bzw. 19,6\% (Hospize) ziehen bei Bedarf einen Kunsttherapeuten hinzu. Weitere Studien zur Versorgungssituation durch komplementäre Therapien liegen aus den USA vor, wo künstlerische Therapien im Gesundheitssystem insgesamt zugenommen haben [38].

Die Angaben zur Häufigkeit kunsttherapeutischer Angebote in der dortigen Palliativversorgung sind jedoch unterschiedlich. Sie schwanken zwischen 22\% [22] und 29\% [41] in einzelnen Staaten (Washington State bzw. Illinois) und den Ergebnissen einer US-weiten Querschnittstudie, der zufolge etwa $1 / 3$ aller Einrichtungen komplementäre oder alternative Therapien anbieten, differenziert in Massage (74\%), Musiktherapie (53\%) oder Kunsttherapie (22\%) [9]. In einem weiteren Versorgungsbericht (State of the Field Report) [38] wurde ermittelt, dass durch künstlerische Angebote allgemein im Gesundheitswesen in den USA auch Kosten reduziert werden können, da die Dauer der Aufenthalte verkürzt werden, die Patienten zum Teil weniger Medikamente brauchen und sie weniger Komplikationen entwickeln.

\section{Praxiserfahrungen im Fokus der Forschung}

Veröffentlichungen zur Kunsttherapie in der Palliativversorgung sind derzeit überwiegend darauf konzentriert, Erfahrungen und Erkenntnisse aus der Praxis aufzuzeigen. Die Überprüfung von Wirkfaktoren und Wirksamkeiten durch empirische Studien befindet sich noch im Anfangsstadium. Es liegen jedoch vielversprechende Ergebnisse aus angrenzenden Anwendungsfeldern wie der Onkologie vor, aus dem circa 90\% der Patienten in der Palliativversorgung stammen $[13,27]$. Allerdings werden in diesen Übersichtsarbeiten und dazugehörigen Primärstudien Palliativpatienten und die Bedeutung der Kunsttherapie für diese spezifische Gruppe meist nicht differenziert dargestellt. Auch eine krankheitsübergreifende Übersichtsarbeit zur Kunsttherapie bei Patienten im Palliativstadium, wie sie z. B. für die Musiktherapie erarbeitet wurde [42], gibt es bisher nicht. Ziel dieses narrativen Reviews ist es, die vorhandenen Veröffentlichungen zur Kunsttherapie in der Palliativversorgung zusammenzufassen, um den Stand der Forschung und Praxis darzustellen sowie darauf aufbauend eine Grundlage für weitere Forschungsfragen und Forschungsvorhaben vorzubereiten.

Insgesamt wurden alle Publikationen ausgewertet, in denen Forschungsergebnisse, praktische Erfahrungen, Therapieansätze und Fallverläufe beschrieben wurden. In diesem Teil I werden zunächst die Methodik der Recherche und Ergebnisse aus Interventionsstudien zusammengefasst. In Teil II (erscheint in Ausgabe 1/2018) werden die Ergebnisse der Auswertung der Praxis- und Erfahrungsberichte ergänzend vorgestellt. 


\section{Kunsttherapie ist seit über 30 Jahren in der Palliativ- versorgung etabliert: In den Leitlinien wird sie als eine von 9 primär nicht-ärztlichen Tätigkeiten in der begleiten- den Behandlung vorgeschlagen.}

\section{Methodische Vorgehensweise}

Für die Bearbeitung der Fragestellung wurde eine systematische Datenbankrecherche durchgeführt, die letzte Aktualisierung fand am 5. November 2016 statt. Gesucht wurde in den Datenbanken PsycInfo, Medline, Pubmed, Cochrane Central, Biosis Previews, CAB Abstracts, Cochrane CDSR, Cochrane Dare, Psyndex, Scisearch. Als Suchbegriffe wurde „art therapy“ über eine freie verbindende Suche (AND) mit den Begriffen „hospice“, „palliativ“, „terminal ill“, „death“, „dying“, und „end of life“ kombiniert. Eingeschlossen wurden alle Artikel, die sich mit einer kunsttherapeutischen Begleitung von Menschen aller Altersklassen mit lebensbedrohlichen, unheilbaren Erkrankungen auseinandersetzen, unabhängig von ihrem Erscheinungsdatum, Inhalt (empirische Studie, Fall- oder Erfahrungsbericht) oder Ursprungsland, sofern sie auf Deutsch, Englisch oder Französisch verfasst sind. Eine Interventionsstudie, deren Volltext nur auf Chinesisch vorliegt, musste ausgeschlossen werden [7]. Aus- geschlossen wurden außerdem Artikel, die sich ausschließlich mit anderen künstlerischen Therapien (Musiktherapie, Tanztherapie, Dramatherapie, Poesietherapie) in der Palliativversorgung beschäftigen, sowie Artikel zur Kunsttherapie bei einzelnen Krankheitsgruppen, z. B. Krebs / Onkologie, Geriatrie oder anderen schweren Erkrankungen, wenn diese nicht eindeutig eine Patientengruppe beinhalteten, deren Krankheits- / Behandlungsstadium als „palliativ“, „Endstadium“ oder „im fortgeschritten Stadium“ definiert wurde.

Auch Publikationen, die sich mit angrenzenden Bereichen wie der Wirkung von Kunstprojekten ohne damit verbundener oder vorhergehende kunsttherapeutische Intervention auseinandersetzen, wurden für diese Übersichtsarbeit ausgeklammert. Der Suchbegriff „art therapy“ führte zudem zu Artikeln über medizinische Prozeduren (ART-antiretrovirale Therapie) oder „State of the art therapy of...", die ebenfalls ausgeschlossen wurden. Auch zahlreiche Publi-

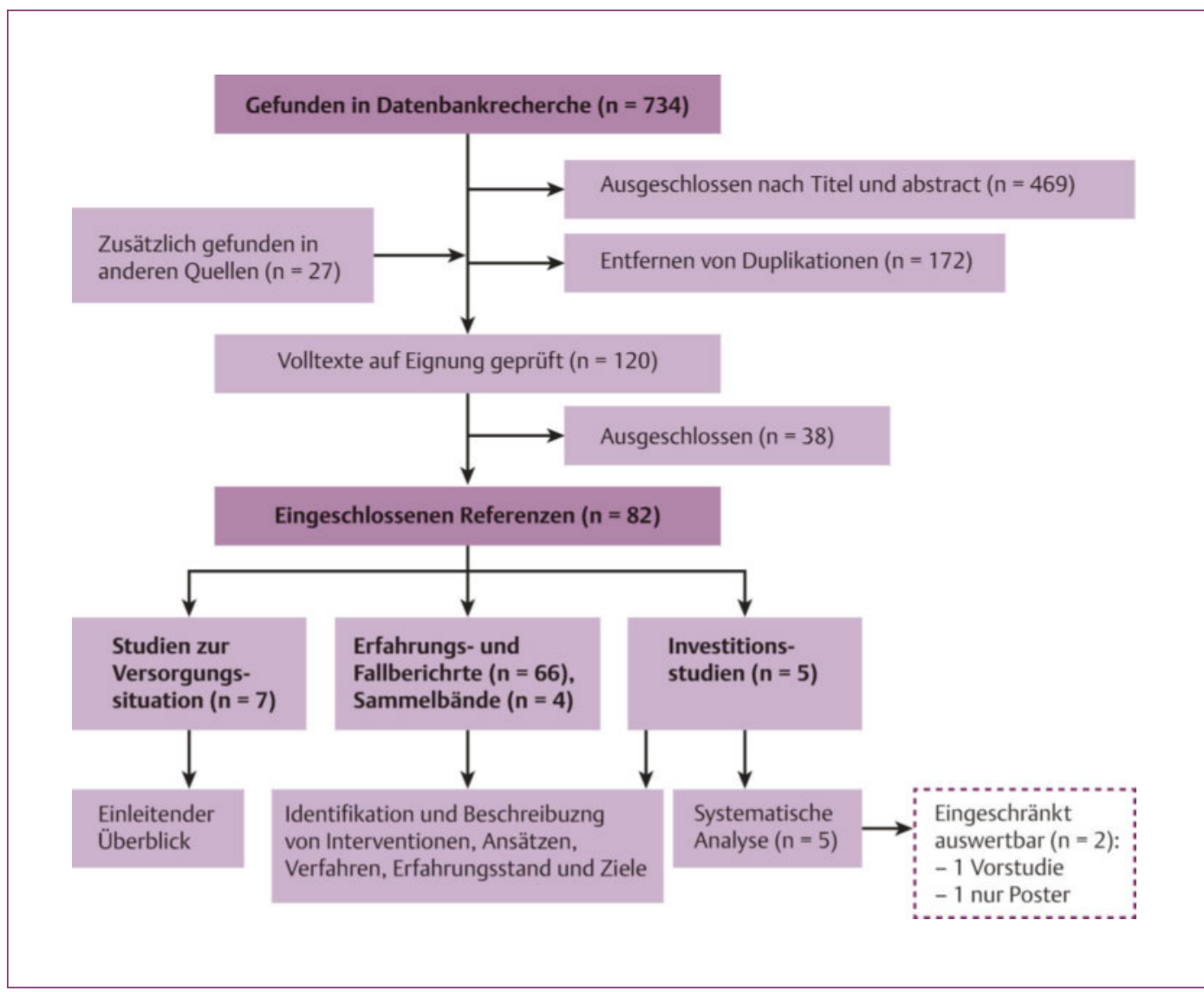




\section{Kunstherapie Wissen zkm}

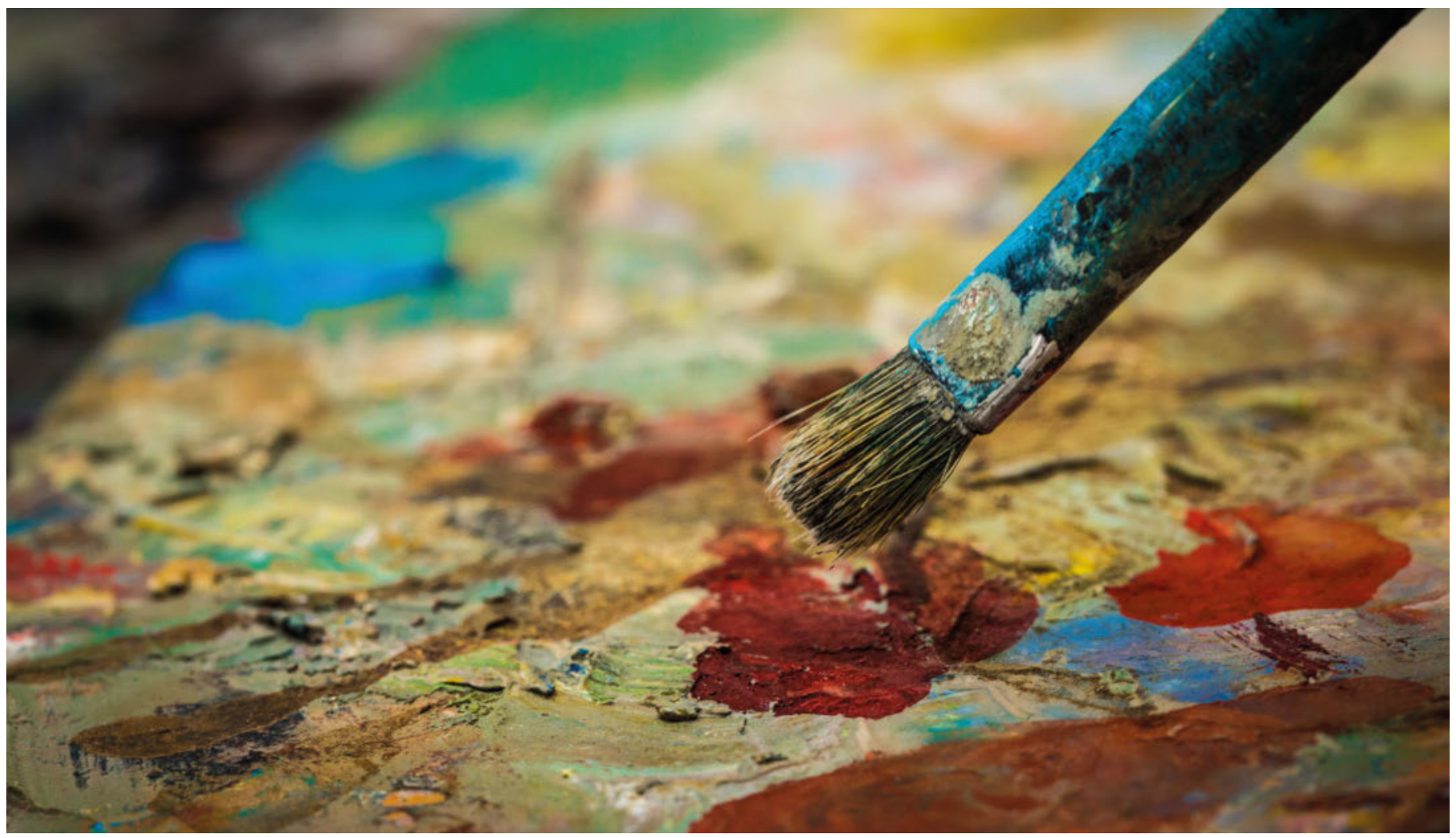

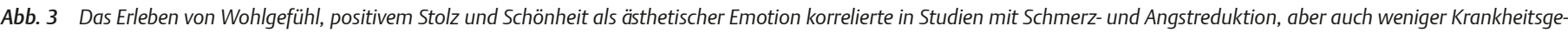
fühl, Traurigkeit und Depression. (c) fotofabrika/stock.adobe

kationen zur Kunsttherapie mit Hinterbliebenen oder Mitarbeitern werden nur beispielhaft erwähnt, jedoch nicht weiter untersucht. Zur Literatur der anderen künstlerischen Therapien (Musiktherapie, Tanztherapie, Dramatherapie, Poesietherapie) liefern Koch und Kollegen [21] eine Übersicht.

Folgende Aspekte werden aus den gefundenen Literaturquellen herausgearbeitet: Forschungsstand und aktuelle Studienlage (Teil I), Interventionen, Ansätzen, Verfahren der Kunsttherapie in der Palliativmedizin, Identifikation und Beschreibung des Erfahrungsstandes und der Ziele aus der Praxis (Teil II in Ausgabe 1/2018).

\section{Ergebnisse}

\section{Literaturquellen zum Thema}

Gefunden wurden 84 nationale und internationale Titel zur Kunsttherapie in der Palliativversorgung im Publikationszeitraum von 1981-2016, davon 5 Interventionsstudien [10, 24-26, 37] aus dem Zeitraum 2008-2016 sowie 7 Studien zur Versorgungssituation durch komplementäre Therapien, in denen auch Kunsttherapie erwähnt wird [8, 9, 17, 22, 38, 41, 44], 66 Erfahrungs- und/oder Fallberichte, 4 Sammelbände, die sich ausschließlich diesem Thema widmen $[6,15,19,32]$. Diese Quellen wurden für unterschiedliche Anteile der Übersichtsarbeit genutzt und durch ergänzende Literatur kontextualisiert und diskutiert (Abb.2).

\section{Forschungsstand}

In 5 prospektiven Interventionsstudien mit zwischen $\mathrm{N}=12$ und $\mathrm{N}=177$ Patienten wurde die Wirkung der kunsttherapeutischen Behandlung bei Patienten in der Palliativversorgung mit verschiedenen methodischen Zugängen untersucht (Tab. 1). Davon liegen zu 3 der Studien umfangreiche Ergebnisberichte vor [10, 25, 26]. 2 weitere sind weniger ausführlich beschrieben [24, 37]. Bei allen Studi- en wurden unterschiedliche Patientengruppen und Erhebungsverfahren eingesetzt, die Methodik und Dauer der Kunsttherapie variierten, eine Kontrollgruppe wurde einmal [10] eingesetzt. Eine systematische Auswertung ist auf dieser Datengrundlage nur eingeschränkt möglich (Tab.1).

Elkis-Abuhoff und Kollegen [10] untersuchten einmalig stattfindende, haptisch ausgerichtete Kunsttherapie bei palliativ betreuten Parkinson-Patienten in einer kontrollierten Studie. Als Outcome wählten sie die grundsätzliche Anwendbarkeit der Intervention und ihre Wirkung auf subjektiv empfundene Beeinträchtigung durch körperliche und psychische Symptome (Brief Symptom Inventory (BSI), Prä-Postvergleich). Sie stellten fest, dass alle Probanden die Möglichkeit der Gestaltung und des Ausdrucks gut nutzten und emotionale, biografische Bezüge entwickeln konnten (Familie, Kindheit, Natur, Hobbies). Bei der Patientengruppe $(\mathrm{N}=21)$ zeigten sich zudem Verbesserungen der somatischen Symptome und des emotionalen Stresses in allen 9 Skalen des Fragebogens. Nach Berechnung der Effektstärken sind davon Reduktionen in 3 Skalen signifikant: Zwanghaftigkeit (O-C: leichte Konzentrations- und Arbeitsstörungen bis hin zur ausgeprägten Zwanghaftigkeit), phobische Angst (PHOB: leichtes Gefühl von Bedrohung bis hin zur massiven phobischen Angst) und Depression (DEP: Traurigkeit bis hin zur schweren Depression).

In allen anderen Studien wurde Kunsttherapie bei Krebspatienten im fortgeschrittenen Stadium untersucht [24-26, 37]. Lin et. al [26] rückten dabei die Wirkungen rezeptiv und aktiv ausgerichteter kunsttherapeutischer Interventionen bei einer Stichprobe von $\mathrm{N}=177$ in den Fokus und ermittelten in strukturierten Interviews mit den Patienten und ihren Familien anhand von Kategorisierungen und Häufigkeitsanalysen unterschiedliche Ausprägungen wesentlicher Erfahrungs- und Wirkbereiche: 70\% fühlten sich nach der 


\section{Charakteristik der Interventionsstudien zur Kunsttherapie in der Palliativversorgung}

\begin{tabular}{|c|c|c|c|c|c|c|c|c|}
\hline Autor & Population & Intervention & Outcomes & Instrumente (Auswertung) ${ }^{*}$ & \multicolumn{4}{|c|}{ Ergebnisse, Effektstärken (Cohen's d)** } \\
\hline \multirow{3}{*}{$\begin{array}{l}\text { Elkis- } \\
\text { Ab- } \\
\text { uhoff } \\
\text { et al. } \\
\text { (2008) } \\
{[10]}\end{array}$} & \multirow{3}{*}{$\begin{array}{l}\mathrm{N}=41: \text { Parkin- } \\
\text { sonpatienten } \\
(\mathrm{N}=22)(16 \mathrm{~m}, \\
7 \mathrm{w}) / \text { Kontroll- } \\
\text { gruppe: } \\
(\mathrm{N}=19) \text { Pfle- } \\
\text { gende u.a. }(12 \\
\mathrm{m}, 7 \text { w) }\end{array}$} & \multirow{3}{*}{$\begin{array}{l}\text { Manipulation } \\
\text { farbiger Knet- } \\
\text { kugeln } \\
1 \text { Sitzung }\end{array}$} & \multirow{3}{*}{$\begin{array}{l}\text { Anzahl und } \\
\text { Intensität der } \\
\text { Symptome } \\
\text { Anwendbar- } \\
\text { keit, Annahme } \\
\text { der Interventi- } \\
\text { on }\end{array}$} & $\begin{array}{l}\text { BSI, vor/nach Intervention } \\
\text { (zweipaariger T-Test) }\end{array}$ & \multicolumn{4}{|c|}{$\begin{array}{l}\text { BSI: } 3 \text { statistisch relevante Symptombereiche: Zwanghaftigkeit (O-C): } \\
d=1,6 \text {, Depression (DEP): } d=1,0 \text {, Phobische Ängstlichkeit (PHO): } d=1,0\end{array}$} \\
\hline & & & & $\begin{array}{l}\text { Strukturierte Interviews Patien- } \\
\text { ten/Kontrollgruppe; Prozess-| }\end{array}$ & \multicolumn{4}{|c|}{$\begin{array}{l}100 \%(N=41) \text { konnten Knete bearbeiten, davon } 73 \% \text { in wiedererkennba- } \\
\text { rer Form, überwiegend }(68,29 \%) \text { mit emotionaler Reaktion }\end{array}$} \\
\hline & & & & $\begin{array}{l}\text { Produktdokumentation (quali- } \\
\text { tative Auswertung, Häufig- } \\
\text { keitsvergleiche) }\end{array}$ & \multicolumn{4}{|c|}{$\begin{array}{l}\text { Geschlechtsspezifische Unterschiede: Patientengruppe ( } N=16 \text { m vs. } 7 \text { w): } \\
\text { Farbwahl (Männer: gelb, blau; Frauen heterogen), zukünftiges Interesse an } \\
\text { Kunsttherapie (Männer: 62,5\%, Frauen: } 100 \% \text { ) }\end{array}$} \\
\hline \multirow{4}{*}{$\begin{array}{l}\text { Lin et } \\
\text { al. } \\
(2012) \\
{[26]}\end{array}$} & \multirow{4}{*}{$\begin{array}{l}\mathrm{N}=177 \\
(105 \mathrm{~m}, 72, \mathrm{w}) \\
\text { Kontrollgrup- } \\
\text { pe: keine }\end{array}$} & \multirow{4}{*}{$\begin{array}{l}\text { Rezeptive und } \\
\text { aktive Kunst- } \\
\text { therapie } \\
1-16 \text { Sitzungen }\end{array}$} & \multirow{4}{*}{$\begin{array}{l}\text { Künstlerischer } \\
\text { Ausdruck } \\
\text { Gefühle, Freu- } \\
\text { de, Gedanken, } \\
\text { Verhalten der } \\
\text { Patienten in } \\
\text { Bezug auf } \\
\text { Kunsttherapie }\end{array}$} & \multirow{4}{*}{$\begin{array}{l}5 \text { Items künstlerischer Aus- } \\
\text { druck: Schönheit, Freude an } \\
\text { der Kunst, Kreativität, aktives } \\
\text { Gestalten, Engagement }{ }^{* * *} \text { - } \\
\text { vor/nach Intervention (T-Test/ } \\
\text { Mann Whitney U Test) } \\
\text { Strukturierte Interviews (quali- } \\
\text { tative Inhaltsanalyse, Häufig- } \\
\text { keitsvergleiche) }\end{array}$} & \multirow{2}{*}{\multicolumn{4}{|c|}{$\begin{array}{l}\text { Veränderung Künstlerischer Ausdruck: } d=1,63 \\
\text { Interviewergebnisse }(\mathrm{N}=177): 70 \% \text { emotional entspannter, } 53,1 \% \text { phy- } \\
\text { sisch besser, } 80 \% \text { Aktivierung positiver Erinnerungen }\end{array}$}} \\
\hline & & & & & & & & \\
\hline & & & & & \multirow{2}{*}{\multicolumn{4}{|c|}{$\begin{array}{l}\text { Keine Unterschiede zwischen Geschlechtern, Unterschiede: bei niedrige- } \\
\text { rem vs. höherem Bildungsabschluss }(2,3 \pm 1,3 \text { vs. } 1,4 \pm 1,3) \text { sowie geringer } \\
\text { vs. ausgeprägter künstlerischer Vorerfahrungen }(1,9 \pm 1,3 \text { vs. } 0,5 \pm 1,2) \text { im } \\
\text { Mittel signifikant größere Verbesserungen des künstlerischen Ausdrucks } \\
(p<0,05)\end{array}$}} \\
\hline & & & & & & & & \\
\hline \multirow{12}{*}{$\begin{array}{l}\text { Lefèv- } \\
\text { re; } \\
\text { Ledoux } \\
\text { et al. } \\
\text { (2016) } \\
{[25]}\end{array}$} & \multirow{12}{*}{$\begin{array}{l}\mathrm{N}=22 \\
(6 \mathrm{~m}, 16 \mathrm{w}) \\
\text { Kontrollgrup- } \\
\text { pe: keine }\end{array}$} & \multirow{12}{*}{$\begin{array}{l}\text { Begleitetes, } \\
\text { freies Arbeiten) } \\
1-10 \text { Sitzungen }\end{array}$} & \multirow{12}{*}{$\begin{array}{l}\text { Symptome } \\
\text { Zusammen- } \\
\text { hang ästheti- } \\
\text { sche Erfahrung } \\
\text { und Symptome }\end{array}$} & \multirow{12}{*}{$\begin{array}{l}\text { ESAS modifiziertt****, } 5 \text { Minu- } \\
\text { ten vor/nach jeder Sitzung } \\
\text { (Wilcoxon Test) } \\
\text { Harmonic Cube (Wohlgefühl, } \\
\text { Stolz, Schönheit }{ }^{* * * * *} \text { ) zur } \\
\text { ästhetischen Erfahrung [110, } \\
\text { 111]: } 5 \text { Minuten nach Kunst- } \\
\text { therapie (Wilcoxon Test, Korre- } \\
\text { lationen: Spearman)t }\end{array}$} & \multirow{2}{*}{\multicolumn{4}{|c|}{$\begin{array}{l}\text { ESAS: Schmerzen: } d=0,40 \text {; Angst: } d=0,62 ; \text { Krankheitsgefühl: } d=0,98 \text {; } \\
\text { Müdigkeit: } d=0,65 ; \text { Traurigkeit: } d=0,74 \text {; Depression: } d=0,76 \text {; Total Dist- } \\
\text { ress (Summe der } 6 \text { Symptome): } d=0,99 \\
\text { Stärke des Zusammenhangs ästhetischer Erfahrung und Symptome (in } \\
\text { Cohen's ds aus Korrelationen errechnet): }\end{array}$}} \\
\hline & & & & & & & & \\
\hline & & & & & & Wohlgefühl & Stolz & Schönheit \\
\hline & & & & & Schmerz & $-0,8065$ & $-1,0247$ & $-1,208$ \\
\hline & & & & & Angst & $-1,0191$ & $-1,065$ & $-0,5811$ \\
\hline & & & & & sich krank & $-1,0679$ & $-0,6475$ & $-0,5452$ \\
\hline & & & & & fuhlen & & & \\
\hline & & & & & Müdigkeit & $-0,3786$ & 0,0038 & 0 \\
\hline & & & & & Traurigkeit & $-0,404$ & $-0,8703$ & $-0,3304$ \\
\hline & & & & & Depression & $-0,6015$ & $-0,8548$ & $-0,345$ \\
\hline & & & & & $\begin{array}{l}\text { Total Dist- } \\
\text { ress }\end{array}$ & $-0,7521$ & $-0,5992$ & $-0,4554$ \\
\hline & & & & & \multicolumn{4}{|c|}{ Keine Unterschiede zwischen den Geschlechtern. } \\
\hline \multirow{3}{*}{$\begin{array}{l}\text { Rhon- } \\
\text { dali et } \\
\text { al. } \\
\text { (2013) } \\
{[37]}\end{array}$} & $\begin{array}{l}\mathrm{N}=12 \text { (weib- } \\
\text { lich) } \\
\text { Krebspatientin- } \\
\text { nen, fortge- }\end{array}$ & \multirow{3}{*}{$\begin{array}{l}\text { Begleitetes, } \\
\text { freies Malen } \\
1 \text { Sitzung }\end{array}$} & \multirow{3}{*}{$\begin{array}{l}\text { Symptome } \\
\text { Machbarkeit } \\
\text { und Annahme, } \\
\text { Einstellung der } \\
\text { Patienten zur } \\
\text { Kunsttherapie }\end{array}$} & \multirow{3}{*}{$\begin{array}{l}\text { ESAS, } 1 \text { Stunde vor/1 Stunde } \\
\text { nach Intervention (Wilcoxon } \\
\text { Test) } \\
\text { Patienteninter-views, } 1 \text { Tag } \\
\text { nach Kunsttherapie (Inhalts- } \\
\text { analyse) }\end{array}$} & \multirow{3}{*}{\multicolumn{4}{|c|}{$\begin{array}{l}\text { ESAS: signifikante Verbesserungen: Schmerzen }(p=0,002) \text {, Fatigue } \\
(p=0,001) \text {, Angst }(p=0,027) \text {, Wohlbefinden }(p=0,015) \text {. Signifikanzniveau: } \\
p<0,05 \\
\text { Interviewergebnisse: wohltuend, entspannende Effekte, „Pause von der } \\
\text { Erkrankung“/Ablenkung von körperlichen Schmerzen, positive soziale } \\
\text { Wirkung (Freunde, Familie, Pfleger) }\end{array}$}} \\
\hline & $\begin{array}{l}\text { schrittenes } \\
\text { Stadium }\end{array}$ & & & & & & & \\
\hline & $\begin{array}{l}\text { Kontrollgrup- } \\
\text { pe: keine }\end{array}$ & & & & & & & \\
\hline \multirow{3}{*}{$\begin{array}{l}\text { Lari- } \\
\text { don et } \\
\text { al. } \\
\text { (2015) } \\
{[24]}\end{array}$} & \multirow{3}{*}{$\begin{array}{l}\mathrm{N}=80 \text { Krebser- } \\
\text { krankungen, } \\
\text { davon } 60 \% \\
(\mathrm{~N}=48) \mathrm{im} \\
\text { palliativen } \\
\text { Stadium } \\
\text { Kontrollgrup- } \\
\text { pe: keine }\end{array}$} & \multirow{3}{*}{$\begin{array}{l}\text { Individualisier- } \\
\text { te Einzelset- } \\
\text { tings } \\
\text { Mindestens } 3 \\
\text { Sitzungen }\end{array}$} & $\begin{array}{l}\text { Symptome } \\
\text { Lebensqualität }\end{array}$ & $\begin{array}{l}\text { Visuelle Analogskala, Single- } \\
\text { Item Messung; vor/nach jeder } \\
\text { Sitzung (Mann-Whitney U Test) } \\
\text { HADS, WHOOOL-BREF bei Ein- }\end{array}$ & $\begin{array}{l}\text { Kurzzeiteffekt } \\
\text { Schmerz: } 41 \% \\
\text { ativ), Reduktic } \\
\text { Ausgangssitua }\end{array}$ & $\begin{array}{l}\text { : signifikante } \mathrm{R} \\
\mathrm{p}<0,001) \text { una } \\
\text { T Fatigue }(20 \% \\
\text { ion). Signifikar }\end{array}$ & $\begin{array}{l}\text { eduktione } \\
\text { hängig vc } \\
\text { bei Pallia } \\
\text { zniveau: } \mathrm{p}\end{array}$ & $\begin{array}{l}\text { gst: } 66 \%(p<0,0001) \text {; } \\
\text { adium (palliativ vs. non-palli- } \\
\text { tienten (schlechtere/müdere } \\
5\end{array}$ \\
\hline & & & $\begin{array}{l}\text { Kurz- und Lang- } \\
\text { zeiteffekte }\end{array}$ & schluss (t0), 3./6. Sitzungen/ & $\begin{array}{l}\text { Langzeiteffekt } \\
\text { über die Zeit, }\end{array}$ & $\begin{array}{l}\text { WHOQOL-B } \\
\text { ine Verbesse }\end{array}$ & $\begin{array}{l}\text { F, HADS } \\
\text { ung bei S }\end{array}$ & $\begin{array}{l}\text { sserungen QoL, Angst (60\%) } \\
\text { z, } 90 \% \text { der Gesamtgruppe }\end{array}$ \\
\hline & & & & BDscriluss & $\begin{array}{l}(\mathrm{N}=80) \text { erlebt } \\
\text { keit auf Positi }\end{array}$ & $\begin{array}{l}\text { n Kunstthera } \\
\text { s, } 89 \% \text { als be }\end{array}$ & $\begin{array}{l}\text { e als Abler } \\
\text { higend/e }\end{array}$ & $\begin{array}{l}\text { g/Lenkung der Aufmerksam- } \\
\text { annend }\end{array}$ \\
\hline & & & Künstlerischer & 5 Items künstlerischer Aus- & Veränderung | & ünstlerischer A & usdruck: $\mathrm{d}$ & \\
\hline Lin et & $N=177$ & Rezeptive und & $\begin{array}{l}\text { Ausdruck } \\
\text { Gefühle, Freu- }\end{array}$ & $\begin{array}{l}\text { druck: Schonneit, Freude an } \\
\text { der Kunst, Kreativität, aktives } \\
\text { Gestalten, Enqagement - vor/ }\end{array}$ & $\begin{array}{l}\text { Interviewerge } \\
\text { sisch besser, } 8\end{array}$ & $\begin{array}{l}\text { nisse }(\mathrm{N}=177) \\
\% \text { Aktivierung }\end{array}$ & $\begin{array}{l}70 \% \text { emo } \\
\text { positiver } \mathrm{E}\end{array}$ & $\begin{array}{l}\text { al entspannter, } 53,1 \% \text { phy- } \\
\text { erungen }\end{array}$ \\
\hline $\begin{array}{l}\text { al. } \\
(2012)\end{array}$ & $\begin{array}{l}\text { (105 m, } 72 \text { w) } \\
\text { Kontrollgrup-- }\end{array}$ & $\begin{array}{l}\text { aktive Kunst- } \\
\text { therapie }\end{array}$ & $\begin{array}{l}\text { de, Gedanken, } \\
\text { Verhalten der }\end{array}$ & $\begin{array}{l}\text { nach Intervention (T-Test/Mann } \\
\text { Whitney U Test) }\end{array}$ & $\begin{array}{l}\text { Keine Untersc } \\
\text { rem vs. höher }\end{array}$ & $\begin{array}{l}\text { iede zwischen } \\
\mathrm{m} \text { Bildungsabs }\end{array}$ & $\begin{array}{l}\text { Jeschlech } \\
\text { hluss }(2,3\end{array}$ & Unterschiede: bei niedrige- \\
\hline [26] & pe: keine & 1-16 Sitzungen & $\begin{array}{l}\text { Patienten in } \\
\text { Bezug auf } \\
\text { Kunsttherapie }\end{array}$ & $\begin{array}{l}\text { Strukturierte Interviews (quali- } \\
\text { tative Inhaltsanalyse, Häufig- } \\
\text { keitsvergleiche) }\end{array}$ & $\begin{array}{l}\text { vs. ausgepräg } \\
\text { Mittel signifik } \\
(p<0.05)\end{array}$ & $\begin{array}{l}\text { r künstlerisch } \\
\text { nt größere Ver }\end{array}$ & $\begin{array}{l}\text { r Vorerfah } \\
\text { pesserung }\end{array}$ & $\begin{array}{l}\text { gen }(1,9 \pm 1,3 \text { vs. } 0,5 \pm 1,2) \text { im } \\
\text { es künstlerischen Ausdrucks }\end{array}$ \\
\hline
\end{tabular}

*BSI: Brief Symptom Inventory; ESAS: Edmonton Symptom Assessment System; WHOQOL-BREF: World Health Organization Quality of Life, Kurzversion; HADS: Hospital Anxiety and Depression Scale.

**Effektstärken wurden im Vergleich der Prä-/Post Daten [32, 33] einmal im Vergleich zur Kontrollgruppe [31] berechnet.

*** Im englischen Original: beauty, art appreciation, creativity, hands-on artwork, and the engagement of creating artwork regularly.

****Bereiche Übelkeit, Schläfrigkeit, Appetitverlust, Atemnot nach Vorstudien [20, 34] als nicht sensitiv für Kunsttherapie weggelassen; „Traurigkeit“ als Symptom ergänzt. ${ }^{* * * * *}$ Im englischen Original: the Good, the Well, the Beautiful. 
Kunsttherapie emotional entspannter, 53,1\% physisch besser, $80 \%$ konnten positive Erinnerungen aktivieren. Ein weiterer Fokus dieser Studie lag auf der Veränderung des künstlerischen Ausdrucks, der anhand von 5 Items (Schönheit, Freude an der Kunst, Kreativität, aktives Gestalten, Engagement) im Prä-Postvergleich überprüft wurde. Nach der Kunsttherapie zeigten sich auf diesen Items Verbesserungen mit großer Effektstärke [26] (Tab.1).

\section{Ablenkung vom Schmerz}

Lefèvre et al. [25] bauten ihre Untersuchung auf der Vorstudie von Rhondali et al. [37] auf, in der die Durchführbarkeit eines MixedMethods-Forschungsansatz, Edmonton Symptom Assessment System (ESAS) und Interviews zur Untersuchung von Kurzzeiteffekten einzelner Kunsttherapiesitzungen bei $\mathrm{N}=12$ Patienten als erfolgreich getestet wurde. Bereits in dieser Vorstudie [37] wiesen erste Befunde auf signifikante Verbesserungen in den Bereichen Schmerz, Fatigue, Angst und Wohlbefinden (ESAS) hin, die durch qualitative Interviewauswertungen ergänzt wurden, in denen die Kunsttherapie als wohltuend, entspannend, Pause/Ablenkung von der Krankheit und den körperlichen Schmerzen sowie als Möglichkeit, die Beziehung zu Freunden, Familien und Pflegern zu verbessern, beschrieben wurde. Lefèvre et al. [25] bestätigen die positiven Vorbefunde in den Symptombereichen des ESAS bei einer Gruppe von N=22 Patienten. Dabei zeigte sich der größte Effekt in der Reduktion des Gesamtwerts des Distress, gefolgt von Verbesserungen in den Bereichen Befindlichkeit, Depression, Traurigkeit, Müdigkeit und Angst. Im Bereich Schmerzen zeigten sich schwach ausgeprägte Effekte, die jedoch signifikant mit Schönheitserfahrungen korrelierten.

\section{Ästhetische Erfahrung verändert sich}

Die Veränderung der ästhetischen Erfahrung wurde in Korrelation zu den einzelnen Symptomen untersucht. Mithilfe einer Skala (010) des Harmonic Cubes $[11,12]$ wurde das Ausmaß des Erlebens von Wohlgefühl während der künstlerischen Gestaltung (GOOD), positiven Stolzes (nahe an der Selbstwirksamkeitserfahrung) (WELL) und Schönheit als ästhetischer Emotion (BEAUTIFUL) während und nach der kunsttherapeutischen Intervention erfasst. Die Ergebnisse zeigten große Effektstärken in den Korrelationen von Wohlgefühl, Stolz und Schönheit mit Schmerzreduktion; Wohlgefühl und Stolz mit Angstreduktion; Wohlgefühl mit Reduktion des Krankheitsgefühls; Stolz mit Reduktion von Traurigkeit und Depression sowie Wohlgefühl mit dem Distress-Gesamtscores. In anderen Kombinationen sind mittlere bis kleinere Effekte erkennbar (Tab. 1).

\section{Messbare Effekte nach einer Sitzung}

Die Effekte auf die Symptome waren nicht abhängig von der Anzahl der kunsttherapeutischen Interventionen (1-10). Das Wohlgefühl änderte sich bereits nach einer Sitzung signifikant. Die Effekte auf Schönheitserleben und Stolz als Dimensionen der ästhetischen Erfahrung zeichneten sich erst nach weiteren Interventionen ab. Es gab keine Geschlechtereffekte. In einer weiteren Studie aus der Onkologie [24] werden die Effekte der Kunsttherapie zwischen Palliativ- und Non-Palliativpatienten differenziert. Untersucht wurde eine Gesamtgruppe von N=80 onkologischen Patienten, davon $60 \%$ im Palliativstadium, die über den Zeitraum von 3 Monaten im Schnitt $7 \pm 4$ Sitzungen Kunsttherapie erhielten. Mit einer „Single-
Item Messung“ wurden Kurzzeiteffekte ermittelt. Hier zeigten sich signifikante Wirkungen auf Symptome und Lebensqualität unabhängig vom Stadium der Erkrankung. Unterschiede gab es nur im Bereich Fatigue, in dem die Verbesserungen bei den Palliativpatienten bei gleichzeitig schlechterer / müderer Ausgangssituation signifikant ausgeprägter waren als bei den Non-Palliativpatienten. Außerdem fanden bei Letzteren mit 8- vs. 5-mal Kunsttherapie durchschnittlich mehr Interventionen statt, als bei den Palliativpatienten. Insgesamt $(\mathrm{N}=80)$ erlebte ein Großteil der Patienten (90\%) die Kunsttherapie als Ablenkung / Lenkung der Aufmerksamkeit auf etwas Positives und $89 \%$ als beruhigend und entspannend. Bei den Langzeiteffekten (Hospital Anxiety and Depression Scale (HADS), World Health Organization Quality of Life, kurz: WHOQOL-BREF) waren die Verbesserungen innerhalb der Gesamtgruppe im Bereich Lebensqualität und Angstreduktion bei $60 \%$ deutlich, nicht aber im Bereich der Schmerzen.

\section{Wirkfaktoren: Bildungsabschluss und Kunstbezug}

In 3 der Studien wurden Unterschiede innerhalb der Gruppen vor und nach der kunsttherapeutischen Intervention differenziert [10, 25, 26]. Eine Studie verglich die Effekte mit den Veränderungen bei einer Kontrollgruppe [10]. Geschlechterdifferenzen zeigten sich nur im Rahmen der letztgenannten Studie bei einer Intervention mit bunten Knetkugeln bei Parkinsonpatienten. Hier gab es bei den Männern deutlichere Farbpräferenzen (blau und gelb), während das Interesse an weiterer Kunsttherapie innerhalb dieser Gruppe bei den Frauen stärker ausgeprägt war [10]. Lin et al. [26] stellten fest, dass Patienten mit niedrigem Bildungsabschluss und geringer künstlerischer Vorerfahrungen stärkere Verbesserungen im ästhetischen Ausdruck erzielten als Patienten mit höherem Bildungsabschluss, ausgeprägten künstlerischen Vorerfahrungen und eingeschränkter positiver Expressivität. Sie fanden ebenso wie Lefévre et al. [25] keine Geschlechts- oder Alterseffekte und auch keine Unterschiede in Abhängigkeit von der onkologischen Erkrankung.

In Tabelle 1 sind die Ergebnisse der 5 vorliegenden Interventionsstudien zusammengefasst.

\section{Diskussion}

Mit diesem ersten Teil der Übersichtsarbeit wurden aus einer umfangreichen Datenbankrecherche alle empirischen Forschungsarbeiten seit 2008 zur Kunsttherapie in der Palliativversorgung zusammenfassend dargestellt. Die qualitativ anhand von strukturierten Patientenbefragungen ermittelten Untersuchungsergebnisse zeigen, dass Patienten trotz ihres fortgeschrittenen Leidens autonom schöpferisch tätig werden wollen, sich mit sich und ihrer Situation auseinandersetzen können und darin eine Entlastung erfahren. Aus Sicht der Patienten können demzufolge durch die Kunsttherapie positive Erinnerungen an persönliche Themen, wie Kindheit, Familie, Natur oder Hobbys geweckt werden [10, 26]. Kunsttherapie wird als emotional entspannend, wohltuend, beruhigend, die Aufmerksamkeit (ab)lenkend und soziale Wirkungen erzeugend erlebt [24, 26, 32]. Damit werden Erfahrungsräume eröffnet, die den Zielsetzungen psychotherapeutischer Interventionen bei Patienten in der Palliativversorgung entsprechen, in denen es um die Reduktion psychosozialer Belastungen und die Aufrechterhaltung von Lebensqualität, Selbstwirksamkeit und Würde geht [28]. In den vorliegenden quantitativen Befunden zeichnet sich ab, dass Kunsttherapie 
grundsätzlich geeignet ist, Belastungen in einzelnen Symptombereichen, wie Depression, Traurigkeit, Angst, Müdigkeit, Fatigue und Schmerz zu reduzieren und Wohlbefinden zu verbessern [10, 24, 25 , 37].

\section{Befunde vorsichtig interpretieren}

Die Befundlage muss jedoch mit Vorsicht interpretiert werden, da die hier vorgesellten Studien nur in Einzelfällen mit hinreichend großen oder kontrollierten Stichproben gearbeitet haben. Sie stehen jedoch in Bezug zu Ergebnissen aus der Kunsttherapie bei unterschiedlichen Stadien onkologischer Erkrankungen, bei denen eine umfangreichere Datenlage vorliegt, die auf eine signifikante Reduktion von Angst und Depression [3, 16, 29, 30, 33, 40], ein geringeres Ausmaß des Erlebens von Stress [14, 29], eine verbesserte Wahrnehmung von Gesundheit und eine Verbesserung der Krankheitsbewältigung [16, 39], Verbesserung der Lebensqualität [29, 36, 39] und Unterstützung der psychischen Gesundheit durch die kreativen, begleitenden Interventionen hinweist [29, 39].

Im Rahmen der Wirkfaktorenforschung ist herauszustellen, dass in 2 Studien einzelne spezifische Aspekte der Kunsttherapie respektive Dimensionen der ästhetischen Erfahrung und des künstlerischen Ausdrucks und ihre Sensitivität im Prä-/Postvergleich mit untersucht wurden $[25,26]$. Daraus geht hervor, dass bei zukünftigen Untersuchungen Bildungsstand, künstlerische Vorerfahrungen und Expressivität der Klienten mitberücksichtigt werden müssen, da sie Einfluss auf die Ergebnisse haben können [26]. Patienten mit niedrigerem Bildungsstand und geringer künstlerischer Vorerfahrung profitierten zumindest in dieser Studie besonders von der Kunsttherapie, möglicherweise, weil sie überrascht sind von einer neuen positiven Erfahrung, die sie noch nicht kannten [26] und damit, bei geringerer Ausgangserwartung an diese Art Therapieangebot, deutlichere Steigerungen des positiven Erlebens möglich sind. Gleichzeitig sprechen die Ergebnisse dafür, dass auch Patienten mit wenig Bezug zur künstlerischen Gestaltung profitieren können und eine Motivation zur Teilnahme an der Kunsttherapie auch bei Hemmungen oder selbstkritischer Haltung der Patienten lohnenswert sein kann.

\section{Wohlgefühl entfaltet sich schnell}

Die Ergebnisse von Lefèvre et al. [25], bei denen Veränderungen spezifischer kunsttherapeutischer Aspekte nicht isoliert, sondern in ihrer Korrelation mit Veränderungen in Symptombereichen untersucht wurden, belegen, dass sich gerade das unmittelbare Wohlgefühl in der ästhetisch-sinnlichen Erfahrung bereits in einzelnen Sitzungen entfalten kann. Dafür sprechen auch die positiven Befunde der anderen Untersuchungen, bei denen nur einzelne Interventionen evaluiert wurden $[10,37]$. Gleichzeitig wird deutlich, dass die Effekte dieser Erfahrung auf die Symptomreduktion verstärkt werden, wenn der Umgang mit künstlerischen Medien durch mehrere Interventionen vertieft werden kann [25]. Kunsttherapie scheint demnach sowohl als einmalige Einheit sinnvoll zu sein, was gerade in der Palliativversorgung von Vorteil ist, da eine längerfristige, mehrmalige Teilnahme an begleitenden Therapiemaßnahmen nicht immer gewährleistet ist. Darüber hinaus wird deutlich, dass der Ausbau der Interventionen in längerfristige bzw. besser verfügbare und regelmäßige Angebote durchaus lohnenswert ist. Mit diesen Ergebnissen ist noch keine evidenzbasierte Grundlage für die

\section{Hinweis zur Limitierung des vorliegenden Reviews}

Limitierung: Das vorliegende Review konzentriert sich auf Befunde zur Kunsttherapie im Palliativbereich unter Verwendung der o.g. Suchbegriffe und Eingrenzungen. Die gefundenen Quellen sind vorwiegend deskriptiv.

Es gibt derzeit nur 5 quantitative, empirische Studien, die hier herausgestellt wurden. Die Befunde dieser Studien sind in ihrer Aussagekraft limitiert, da sie durch verschiedene Untersuchungs- und Interventionsmethoden nur bedingt vergleichbar sind, es sich um Studien mit relativ kleinen Untersuchungsgruppen handelt, bei 4 Studien keine Kontrollgruppe vorhanden war und keine der Studien multizentrisch war. Effektstärken konnten demzufolge nur bei einer Studie im Vergleich mit der Kontrollgruppe [10] und bei 2 weiteren Studien auf Basis eines Prä-/Postvergleiches berechnet werden $[25,26]$. Diese sind jedoch in der Regel ermutigend, mit oftmals großen positiven Veränderungen hinsichtlich Lebensqualität und Symptomreduktion.

Sinnvoll sind weitere Recherchen, bei denen nicht nur Studien zur Onkologie, sondern auch Studien zu anderen progredienten Erkrankungen einbezogen und zu den hier ermittelten Ergebnissen in Bezug gesetzt werden, z. B. kunsttherapeutische Untersuchungen zu neurodegenerativen Erkrankungen wie Demenz, Morbus Parkinson oder Multipler Sklerose.

Legitimation der kunsttherapeutischen Praxis geschaffen, sondern es wird eine wichtige Grundlage für die weitere Erforschung der kunsttherapeutischen Wirkfaktoren aufgezeigt, die bisher auch in anderen Anwendungsfeldern nur beschrieben, nicht aber systematisch untersucht wurden [18]. Hier wäre auch ein interdisziplinärer Austausch zwischen den künstlerischen Therapien sinnvoll, um die Bedeutung verschiedener schöpferischer, kreativer Tätigkeiten transdisziplinär verbindend oder differenzierend zu ermitteln [2] [42] und ihren Stellenwert in multidisziplinären Behandlungsansätzen zu untersuchen.

Abschließend ist festzuhalten, dass Kunsttherapie gerade in der Palliativversorgung besondere Möglichkeiten der therapeutischen Begleitung anbietet, durch die wesentliche Zielsetzungen im Rahmen der ganzheitlich ausgerichteten Gesamtbehandlung erfüllt werden können. Allerdings wird sie noch wenig genutzt, wie die Studien zur Versorgungssituation verdeutlichen. Es bedarf weiterer praktischer und wissenschaftlicher Aufbau- und guter Öffentlichkeitsarbeit, um auf das Potenzial der Kunsttherapie aufmerksam zu machen und sie als festen therapeutischen Bestandteil in der Palliativmedizin, sowohl stationär als auch ambulant, weiter zu untersuchen und zu etablieren. Hierfür liefern die Ergebnisse dieses Reviews erste Anhaltspunkte. 
Interessenkonflikt: Die Autoren erklären, dass keine wirtschaftlichen oder persönlichen Verbindungen bestehen.

\section{Online zu finden unter}

http://dx.doi.org/10.1055/s-0043-121402

\section{Literatur}

1 Archer S, Buxton S, Sheffield D. The effect of creative psychological interventions on psychological outcomes for adult cancer patients: a systematic review of randomised controlled trials. Psycho-Oncology 2015; 24 (1): 1-10

2 Archer, S., Buxton, S., \& Sheffield, D. The effect of creative psychological interventions on psychological outcomes for adult cancer patients: a systematic review of randomized controlled trials. Psychooncology 2015; 24 (1): $1-10$

3 Bar-Sela G, Atid L, Danos S et al. Art therapy improved depression and influenced fatigue levels in cancer patients on chemotherapy. Psycho-Oncology 2007; 16 (11): 980-984

$\overline{4}$ Biskup A, Fritze W, Jancke $G$ et al. „Dass man als Mensch behandelt wird, nicht als Stück". Die Hospiz Zeitschrift 2011; 13 (48): 24-29

5 Boehm K, Cramer H, Staroszynski T et al. Arts therapies for anxiety, depression, and quality of life in breast cancer patients: a systematic review and meta-analysis. Evid Based Complement Alternat Med 2014; 2014: 103297

$\overline{6}$ Bolton G, Hrsg. Kunst und Kreativität in der Palliative Care. Bern: Hans Huber; 2008

7 Chen L-C, Ho C-C. Art therapy: The walking-stick of life for terminal-ill patients. The Archive of Guidance \& Counseling 2009; 31 (2): 69-91

8 Conrad C, Muenstedt K, Micke $O$ et al. Survey of members of the German Society for Palliative Medicine regarding their attitudes toward complementary and alternative medicine for cancer patients. Journal of Palliative Medicine 2013; 16 (8): 822-824

9 Dain AS, Bradley EH, Hurzeler R et al. Massage, Music, and Art Therapy in Hospice: Results of a National Survey. Journal of Pain and Symptom Management 2015; 49 (6): 1035-1041

$\overline{10}$ Elkis-Abuhoff DL, Goldblatt RB, Gaydos M et al. Effects of Clay Manipulation on Somatic Dysfunction and Emotional Distress in Patients With Parkinson's Disease. Art Therapy 2008; 25 (3): 122-128

$\overline{11}$ Forestier R. Tout savoir sur l'art occidental. Lausanne, Paris: Favre; 2004

$\overline{12}$ Forestier R. Tout savoir sur l'art-thérapie. Lausanne: Favre; 2009

$\overline{13}$ Gerhard C. Praxiswissen Palliativmedizin: Konzepte für unterschiedlichste palliative Versorgungssituationen. Stuttgart, New York: Georg Thieme Verlag; 2015

$\overline{14}$ Glinzak L. Effects of Art Therapy on Distress Levels of Adults with Cancer: A Proxy Pretest Study. Art Therapy: Journal of American Art Therapy Association 2016; 33 (1): 27-34

$\overline{15}$ Gruber H, Reichelt S, Hrsg. Kunsttherapie in der Palliativmedizin. Berlin: EB Verlag; 2016

$\overline{16}$ Grulke N, Bailer H, Stähle S et al. Evaluation eines maltherapeutischen Angebots für onkologische Patienten in einem Akutkrankenhaus: Eine Pilotstudie. Musik-, Tanz- und Kunsttherapie 2006; 17 (1): 21

$\overline{17}$ Heusser P, Braun SB, Ziegler R et al. Palliative inpatient cancer treatment in an anthroposophic hospital: I. Treatment patterns and compliance with anthroposophic medicine. Forsch Komplementmed 2006; 13 (2): 94-100

$\overline{18}$ Hilleke T, Wilker FW. Ein heuristisches Wirkfaktorenmodell der Musiktherapie. Verhaltenstherapie \& Verhaltensmedizin 2007; 28 (1): 62-85

$\overline{19}$ Jarrett L, Hrsg. Creative Engagement in Palliative Care. New Perspectives on user involvment. Oxford, New York: Radcliffe Publishing; 2007

$\overline{20}$ Kern M, Jakob-Krieger C. »Immer wieder knallt es bei uns ...«. Spannungen (Reizbarkeit, Streitigkeiten) zwischen den Berufsgruppen als Belastungssymptome. In: Müller M, Pfister D, Hrsg. Wie viel Tod verträgt das Team?: Belastungs- und Schutzfaktoren in Hospitzarbeit und Palliativmedizin. Göttingen: Vandenhoeck \& Ruprecht GmbH \& Co KG; 2013: 172-180 $\overline{21}$ Koch S, Gruber H, Kortum R et al. Künstlerische Therapien in der Palliativversorgung - Ein evidenzbasierter Überblick. Hospiz Zeitschrift 2016; (1)

$\overline{22}$ Kozak LE, Kayes L, McCarty R et al. Use of complementary and alternative medicine (CAM) by Washington State hospices. Am J Hosp Palliat Care 2008; 25 (6): 463-468

$\overline{23}$ Lachner J. Bedürfnisse von schwerstkranken und sterbenden Menschen eine qualitative Erhebung in Palliativeinrichtungen. Psychologie in der Medizin 2005; 16 (1): 18-24

$\overline{24}$ Laridon-Valentini F, Mouawad R, Mateescu C, et al. Impact of art therapy on the quality of life of cancer patients: the Salpetriere hospital experience: Poster Congress 2015

$\overline{25}$ Lefevre C, Ledoux M, Filbet M. Art therapy among palliative cancer patients: Aesthetic dimensions and impacts on symptoms. Palliat Support Care 2016; 14 (4): 376-380

$\overline{26}$ Lin M-H, Moh S-L, Kuo Y-C et al. Art therapy for terminal cancer patients in a hospice palliative care unit in Taiwan. Pall Supp Care 2012; 10 (01): 51-57

$\overline{27}$ Lindena G, Woskanjan S, Fahland R. HOPE Bericht. Im Internet: www.hopeclara.de/download/\%20HOPE_2010.pdf; Stand: 20.07.2016

$\overline{28}$ Mehnert A. Psychotherapie in der palliativen Versorgung. Psychother Psychosom Med Psychol 2015; 65 (9-10): 387-96

$\overline{29}$ Monti DA, Peterson C, Kunkel EJS et al. A randomized, controlled trial of mindfulness-based art therapy (MBAT) for women with cancer. Psycho-Oncology 2006; 15 (5): 363-373

$\overline{30}$ Nainis N, Paice JA, Ratner J et al. Relieving Symptoms in Cancer: Innovative Use of Art Therapy. Journal of Pain and Symptom Management 2006; 31 (2): 162-169

$\overline{31}$ Öster I, Svensk A-C, Magnusson E et al. Art therapy improves coping resources: a randomized, controlled study among women with breast cancer. Palliat Support Care 2006; 4 (1): 57-64

$\overline{32}$ Pratt M, Wood MJM, Hrsg. Art therapy in palliative care. London: Routledge; 1998

$\overline{33}$ Puig A, Lee SM, Goodwin L et al. The efficacy of creative arts therapies to enhance emotional expression, spirituality, and psychological well-being of newly diagnosed Stage I and Stage II breast cancer patients: A preliminary study. The Arts in Psychotherapy 2006; 33 (3): 218-228

$\overline{34}$ Radbruch L, Aulbert E, Nauck F. Grundlagen der Palliativmedizin. Definition, Entwicklung und Ziele. In: Aulbert E, Nauck F, Radbruch L, Hrsg. Lehrbuch der Palliativmedizin. Stuttgart; 2012: 1-12

$\overline{35}$ Radbruch L, Nauck F, Sabatowski R. Was ist Palliativmedizin? Im Internet: www.dgpalliativmedizin.de/images/stories/Was_ist_Palliativmedizin_Definitionen_Radbruch_Nauck_Sabatowski.pdf; Stand: 29.03.2016

$\overline{36}$ Reynolds F, Prior $\mathrm{S}$. The role of art making in identity maintenance: case studies of people living with cancer. European Journal of Cancer Care 2006; 15 (4): 333-341

$\overline{37}$ Rhondali W, Lasserre E, Filbet M. Art therapy among palliative care inpatients with advanced cancer. palliative medicine 2013; 27 (6): 571-572

$\overline{38}$ State of the Field Commitee. State of the Field Report: Arts in Healthcare/ 2009. Society for the Arts in Healthcare. 2009

$\overline{39}$ Svensk A-C, Oster I, Thyme KE et al. Art therapy improves experienced quality of life among women undergoing treatment for breast cancer: a randomized controlled study. European Journal of Cancer Care 2009; 18 (1): 69-77

$\overline{40}$ Thyme KE, Sundin EC, Wiberg B et al. Individual brief art therapy can be helpful for women with breast cancer: a randomized controlled clinical study. Palliat Support Care 2009; 7 (1): 87-95

$\overline{41}$ Van Hyfte G], Kozak LE, Lepore M. A survey of the use of complementary and alternative medicine in Illinois hospice and palliative care organizations. American Journal of Hospice \& Palliative Medicine 2014; 31 (5): 553-561

$\overline{42}$ Warth M, Koenig J, Keßler J et al. Musiktherapie in der palliativmedizinischen Versorgung. Gegenwärtiger Stand und aktuelle Entwicklungen. Musiktherapeutische Umschau 2014; 35 (4): 261-274 
$\overline{43}$ Weglage G. Leben auf Zeit: Anpassungsstrategien palliativ betreuter Menschen. Frankfurt (Main): Marbuse Verlag; 2014

$\overline{44}$ Wood MJM, Low J, Molassiotis A et al. Art therapy's contribution to the psychological care of adults with cancer: A survey of therapists and service users in the UK. International Journal of Art Therapy 2013; 18 (2): 42-53

$\overline{45}$ Wood MJM, Molassiotis A, Payne S. What research evidence is there for the use of art therapy in the management of symptoms in adults with cancer? A systematic review. Psycho-Oncology 2011; 20: 135-145

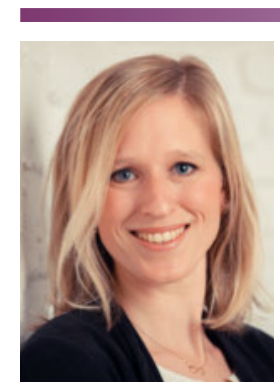

Dr. Ria Kortum

Deutsche Kinderkrebsstiftung

Adenauerallee 134

53113 Bonn

r.kortum@kinderkrebsstiftung.de

Dr. Ria Kortum arbeitete als Kunsttherapeutin auf der kinderkardiologischen Station und in der Ambulanz im Herzzentrum der Universitätsklinik zu Köln sowie als wissenschaftliche Mitarbeiterin im Fachbereich künstlerische Therapien und Therapiewissenschaft an der Alanus Hochschule für Kunst und Gesellschaft. Seit Juli 2017 ist sie Projektreferentin bei der Deutschen Kinderkrebsstiftung.

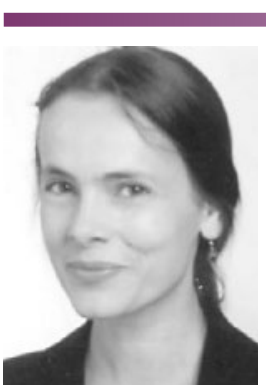

Prof. Dr. Sabine Koch

Alanus Hochschule

Villestraße 3

53347 Alfter bei Bonn

sabine.koch@alanus.edu

Prof. Dr. Sabine Koch ist Psychologin, Tanz- und Bewegungstherapeutin. Sie leitet das Forschungsinstitut für Künstlerische Therapien (RIArT) an der Alanus Hochschule Alfter und das Masterstudium Tanz- und Bewegungstherapie der SRH Hochschule Heidelberg.

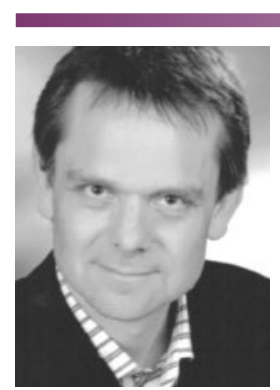

Prof. Dr. Harald Gruber

Alanus Hochschule

Villestraße 3

53347 Alfter bei Bonn

harald.gruber@alanus.edu

Prof. Dr. Harald Gruber arbeitete in unterschiedlichen medizinischen-therapeutischen Einrichtungen und war dort unter anderem an der Entwicklung und Durchführung von kunsttherapeutischen Forschungsprojekten beteiligt. Seit 2007 ist er Professor für Kunsttherapie und Fachbereichsleiter des Fachbereichs Künstlerische Therapien \& Therapiewissenschaft an der Alanus Hochschule für Kunst und Gesellschaft Alfter. 